\title{
Pre- and Post-operative Cerebral Blood Flow Changes in Subarachnoid Haemorrhage
}

\author{
J. M. Mountz ${ }^{1}$, J. E. McGillicuddy ${ }^{2}$, M. W. Wilson ${ }^{3}$, S. P. Bartold ${ }^{1}$, and E. M. Siegal ${ }^{3}$ \\ The University of Michigan Medical Center, ${ }^{1}$ Division of Nuclear Medicine, ${ }^{2}$ Department of Neurosurgery and ${ }^{3}$ The University of Michigan \\ Medical School, Ann Arbor, Michigan U.S.A.
}

\section{Summary}

Assessment of cerebral perfusion on patients with subarachnoid haemorrhage (SAH) in the Neurologic Intensive Care Unit is difficult since nuclear medicine imaging modalities capable of measuring cerebral blood flow (CBF) are not generally available. We performed 101 quantitative ( $\mathrm{ml} / 100 \mathrm{~g}$-min) bedside CBF measurements on 40 individual patients to correlate $\mathrm{SAH}$ grade with $\mathrm{CBF}$ and to assess the effect of surgical intervention on CBF. Global CBF ( $\left.\mathrm{G}_{m} \mathrm{CBF}\right)$ and bihemispheric $\mathrm{CBF}$ (B-CBF) asymmetry were correlated with the grade of $\mathrm{SAH}$ pre- and post-operatively.

Data analysis showed that pre-operative patients with low grade SAH (Hunt and Hess grades 0 to 2) had higher mean G-CBF values $[44.2 \pm .71]$ than those with high grade SAH (Hunt and Hess grades 3 to 4): [mean G-CBF $=34.1 \pm 1.7]$. Post-surgery there was a significant improvement in G-CBF; CBF increased [5.3 1.07] in the group of patients with low grade SAH. Patients with high grade SAH showed no significant improvement in their G-CBF during the first week post-operatively compared to pre-operative values.

We conclude that portable units capable of measuring bedside $\mathrm{CBF}$ values are useful in monitoring CBF changes in patients with SAH. Patients with low grade SAH have G-CBF within normal limits both pre-operatively and post-operatively, with a statistically significant increase in CBF during two weeks post-operatively. $\mathrm{Pa}$ tients with high grade SAH show no significant increase in CBF one week post-operatively compared to their pre-operative measures.

Keywords: Cerebral blood flow; subarachnoid haemorrhage; ${ }^{133}$ Xenon; cerebrograph.

\section{Introduction}

Subarachnoid haemorrhage $(\mathrm{SAH})$ as a sequela of aneurysmal rupture ${ }^{2,10}$ frequently leads to severe neurologic impairment ${ }^{8,17}$. Patients with this condition require aggressive management in the neurologic intensive care unit (NICU). Although transmission computed tomograpy (CT) may demonstrate SAH or intracerebral haemorrhage, generally it cannot provide physiologic information concerning cerebral blood flow $(\mathrm{CBF})^{1}$. After admission to the NICU, further evaluation of $\mathrm{CBF}$ by radiographic imaging modalities such as Xenon CT, Single Photon Emission Computed Tomography (SPECT), or Positron Emission Tomography (PET) becomes difficult due to the difficulties in patient transportation to the scanning areas ${ }^{5,6,12}$. Measurement of CBF at the bedside can provide useful prognostic and diagnostic information and eliminate the need for moving unstable patients. There have been numerous publications relating CBF with the clinical grade of SAH 14, 15, 19, 20, and prognosis of initial CBF measurement with clinical course ${ }^{3}$. The effect of aneurysmal surgery on bedside CBF measurements in a large series of patients followed longitudinally over time is presented in this report.

In order to determine the correlation between bedside CBF measurements and the SAH grade as well as to assess the effect of aneurysmal surgery on CBF, we performed serial studies on patients during their preand post-operative course in the NICU. We employed the ${ }^{133}$ Xenon clearance technique ${ }^{3,11,16,18,22}$, obtaining a total of 101 measurements on 40 individual patients at the bedside to determine global and bihemispheric CBF measurements. We hypothesized that higher SAH grades should correlate with lower global CBF(G$\mathrm{CBF}$ ) values and greater bihemispheric $\mathrm{CBF}(\mathrm{B}-\mathrm{CBF})$ asymmetry due, in part, to a greater degree of cerebral artery vasospasm.

\section{Methods}

Patients were selected for the study over a three year period. Since a non-invasive portable CBF measurement system was utilized, nearly all patients with an aneurysm could be examined, and the results therefore reflect a random selection of patients during this 
time period. A total of 101 bedside CBF determinations were performed on 40 patients admitted with SAH secondary to aneurysmal rupture (26 females, 14 males: age range $26-78$ years, mean $=54$ ) utilizing a 10 probe detector system (Novo Diagnostics 10 a Cerebrograph) ${ }^{21}$. Five scintillation detectors were placed adjacent to the head over each hemisphere, surrounding the Sylvian fissure superiorly and inferiorly. Each bank of detectors was fixed in position in a adjustable frame which permitted reproducible positioning on each patient by reference to the external auditory canal and the lateral canthus ${ }^{11}$. Serial CBF values were obtained over the course of the patient's hospitalization (total number of studies per patient ranged between 1 and 6, length of hospital stay ranged between 1 and 52 days). Each patient was injected with approximately $30 \mathrm{mCi}$ of ${ }^{133}$ Xenon dissolved in sterile saline and the arterial input curve to the brain was determined by measuring the expired alveolar ${ }^{133}$ Xenon gas concentrations. The brain counts and the alveolar gas concentrations were deconvolved utilizing the Novo 10 a Cerebrograph on board computer to yield average CBF values (M2 model, $\mathrm{CBF}-15)$ in $\mathrm{ml} / 100 \mathrm{gm}-\mathrm{min}^{21}$. Utilization of this method measures both fast CBF and slow cerebral blood flow compartments and virtually eliminates components of flow activity from the scalp ${ }^{16}$. Count rates were statistically adequate, with each brain detector recording between 1500 and 3000 counts per 5 second interval at peak cerebral uptake ${ }^{21}$. All global and hemispheric $\mathrm{CBF}$ values were corrected to a $\mathrm{pCO}_{2}$ of 40 torr, using the equation [CBF corrected $=\mathrm{CBF}$ uncorrected $\left.+1.8\left(40-\mathrm{pCO}_{2}\right)\right]^{4}$. An asymmetry index was calculated by the formula $|R-L| \div[(R+L) \times .5]$, where $R$ and $\mathrm{L}$ represent the $\mathrm{CBF}$ values of the right and left cerebral hemispheres respectively, obtained by averaging the CBF values from the five scintillation detectors over each hemisphere.

The initial CBF study and each subsequent follow-up study was performed after the following clinical data was obtained: age and sex of patient; grade of SAH (Hunt and Hess); Glasgow Coma score"; mental status; blood pressure; heart rate; hematocrit; $\mathrm{PaCO}_{2}$; extent and duration of hypervolemic therapy; medical management requirements; location of aneurysm; and side of craniotomy. When possible, a computed tomographic scan was also performed.

Patient management consisted of hypervolemic therapy and agents to reduce cerebral oedema (Mannitol and Decadron). The patients entered into the study had normal blood pressure, $\mathrm{PaCO}_{2}$ values, and hematocrits at or above the lower limits of normal. Patients studied had approximately $50 \%$ right and $50 \%$ left frontoparietal craniotomies for surgical aneurysm clipping. The aneurysms were predominantly located in the junction of the internal carotid artery and Circle of Willis, anterior communicating artery (predominantly at its branches), anterior cerebral artery (predominantly at it branches), and middle cerebral artery (predominantly at its branches). No posterior aneurysmal patients were studied.

In order to compare G-CBF values and B-CBF asymmetry longitudinally pre- and post-operatively, patients were combined into two groups according to the Hunt and Hess scale. The good neurologic class corresponded to Hunt and Hess grades 0 to 2 (number of studies $=54$ ), and the poor neurologic class corresponded to Hunt and Hess grades 3 and 4 (number of studies $=47$ ). To compare pre and post surgical CBF values patients were placed in time bin intervals of 0 to 3 days pre-operatively ( $n=19$ studies, 17 patients), 3 to 7 days pre-operatively ( $\mathrm{n}=14$ studies, 8 patients), and 7 to 14 days pre-operatively ( $n=15$ studies, 10 patients) and 0 to 3 days post-surgery ( $\mathrm{n}=24$ studies, 24 patients), 3 to 7 days post-surgery $(n=15$ studies, 13 patients), and 7 to 14 days post-surgery $(n=14$ studies, 8 patients). Data analysis was performed using the Students T-test, and errors are expressed as standard errors of the mean (SEM) unless otherwise stated.

\section{Results}

The results show a decrease in G-CBF with grade of $\mathrm{SAH}$, with pre-operative low-grade $\mathrm{SAH}$ demonstrating higher G-CBF $[$ mean $=44.1 \pm .7]$ than preoperative high-grade SAH [mean $=34.1 \pm 1.7$ ], $\mathrm{P}<.05$. Post-operatively, low-grade $\mathrm{SAH}$ G-CBF $[$ mean $=49.5 \pm .37]$ was also significantly different compared with post-operative high-grade SAH G-CBF $[$ mean $=37.7 \pm .39], \mathrm{P}<.01$ (Fig. 1). CBF in patients with low-grade SAH showed on average significant improvement post-operatively [mean $=49.5 \pm .37$ ] during the first 2 weeks compared with pre-operative studies [mean $=44.1 \pm .7], \mathrm{P}<.05$ (Fig. 1). Comparing $\mathrm{CBF}$ in patients with high-grade $\mathrm{SAH}$, there was no significant difference between G-CBF pre-operatively $[$ mean $=34.1 \pm 1.7]$ and one week post-operatively $[$ mean $=37.7 \pm .39], \mathrm{P}>.05$ (Fig. 1).

Pre-operatively, patients with low-grade SAH had significantly lower differences in CBF percent asymmetry $[3.6 \pm .2]$ as compared to their post-operative

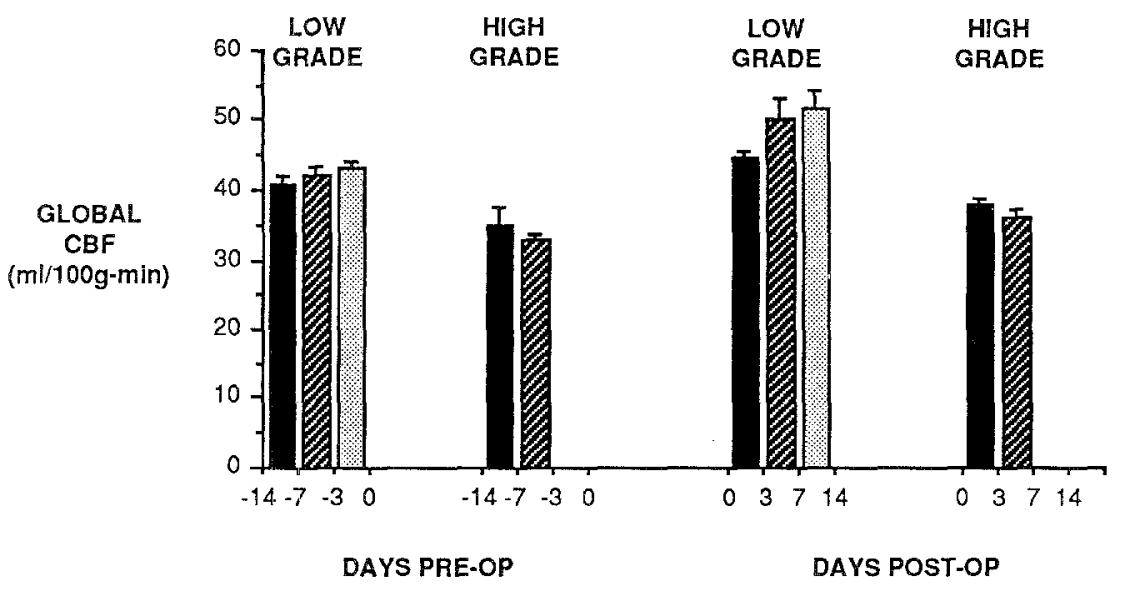

Fig. 1. G-CBF, corrected to 40 torr, for lowand high-grade $\mathrm{SAH}$ at time intervals since ictus pre-surgery or at time intervals postsurgery. On average, patients with low grade SAH showed increased CBF post-surgery compared to pre-surgery. Time bins absent of data reflect the fact that no patients received a $\mathrm{CBF}$ examination during these intervals for the particular grade of SAH 


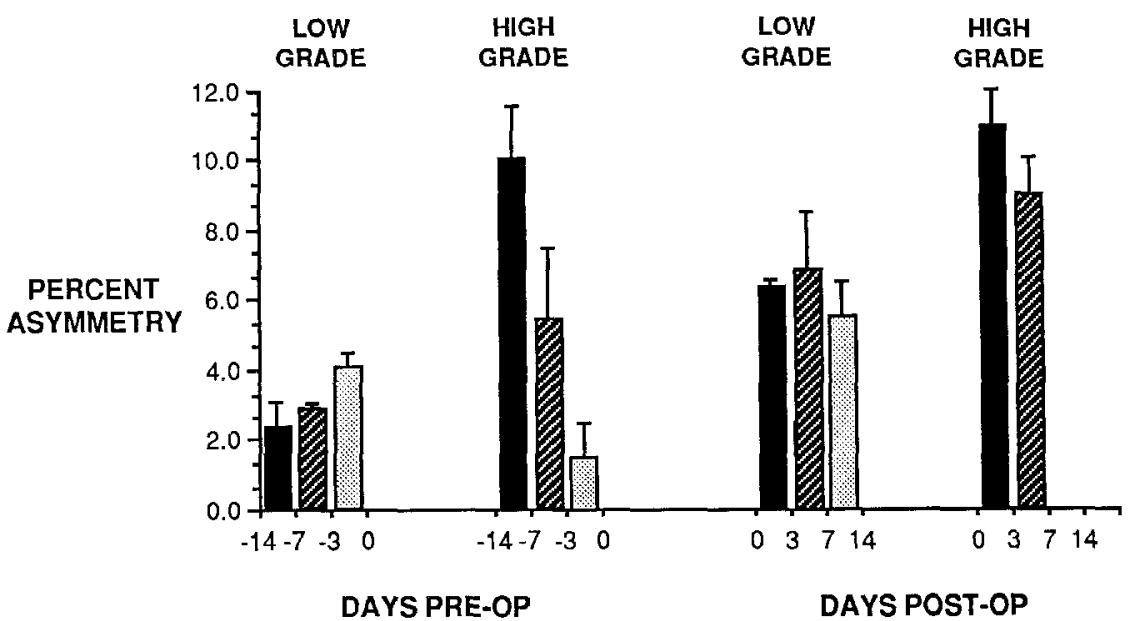

Fig. 2. Percent asymmetry for low- and high-grade SAH at time intervals since ictus pre-surgery or at time intervals post-surgery. Patients with low-grade SAH pre-surgery had low B-CBF asymmetry values, while post-surgery low-grade SAH patients and patients with high-grade SAH had abnormally high B-CBF asymmetry
CBF percent asymmetry values $[6.6 \pm 1.5], \mathrm{P}<.01$ (Fig. 2). Post-operatively, patients with high-grade $\mathrm{SAH}$ showed no significant difference in CBF asymmetry during the first week compared with pre-operative values ( $\mathrm{P}>.05)$ (Fig. 2).

\section{Discussion}

Previous studies of SAH have demonstrated a decline in G-CBF values with higher grades of $\mathrm{SAH}^{9-12}$. There have also been reports correlating prognosis of SAH grade, extent of cerebral vasospasm, and neurologic deficit ${ }^{14}$ using the ${ }^{133}$ Xenon clearance technique. Other studies assessed the cerebral blood flow patterns pre- and post-operatively and found low flows to be associated with poorer clinical grades, with improvement post surgery ${ }^{13,23}$. These reports are in agreement with our findings.

In our study, emphasis was extended on post-operative changes of G-CBF using a portable CBF measurement device which could be brought to the patients bedside in the NICU within minutes of the requested examination. The determination of G-CBF was objectively assessed at the bedside utilizing the ${ }^{133}$ Xenon clearance technique. The results of the bedside G-CBF measures reported here are in agreement with prior studies performed in the radiology department ${ }^{9,12,15}$. Our results support the accuracy of bedside CBF measures, thus avoiding transportation from the NICU to the radiology department. The results demonstrate that patients with low-grade SAH show significant increases in G-CBF during the first two weeks post-operatively. The data also show that patients with high-grade $\mathrm{SAH}$ have low G-CBF values and high B-CBF asymmetry values. These latter patients only showed a modest trend to increase G-CBF during the first week postsurgery as compared to pre-surgery.
The use of bedside CBF measurements in the NICU is a valid and useful method of quickly and repeatedly assessing CBF in patients with subarachnoid haemorrhage and to determine the post-operative changes of $\mathrm{CBF}$ in these patients.

\section{Acknowledgements}

This work was supported, in part, by a grant from the Commonwealth Foundation through the National Medical Fellowships Incorporated.

\section{References}

1. Bonita R, Thomson S (1985) Subarachnoid haemorrhage: epidemiology, diagnosis, management, and outcome. Stroke 16(4): 591-594

2. Chyatte D, Fode NC, Sundt TM (1988) Early versus later intracranial aneurysm surgery in subarachnoid haemorrhage. $J$ Neurosurg 69: 326-331

3. Geraud G, Tremoulet M, Guell A, Bes A (1984) The prognostic value of noninvasive CBF measurement in subarachnoid haemorrhage. Stroke 15: 301-305

4. Grubb RL, Raichle ME, Eichling JO, Ter-Pogossian MM (1974) The effects of changes in $\mathrm{PaCO}_{2}$ on cerebral blood volume, blood flow, and vascular mean transit time. Stroke 5: 630-639

5. Hackney DB, Lesnick JE, Zimmerman RA, Grossman RI, Goldberg HI, Bilaniuk LT (1986) MR identification of bleeding site in subarachnoid haemorrhage with multiple intracranial aneurysms. J Comput Assist Tomogr 10 (5): 878-880

6. Hayman LA, Pagani JJ, Kirkpatrick JB, Hinck VC (1989) Pathophysiology of acute intracerebral and subarachnoid haemorrhage: applications to MR imaging. AJR 153: 135-139

7. Hunt WE, Hess RM (1968) Surgical risk as related to time of intervention in the repair of intracranial aneurysms. J Neurosurg 28: $14-20$

8. Inagawa T, Yamamoto M, Kamiya K, Ogasawara H (1988) Management of elderly patients with aneurysmal subarachnoid haemorrhage. J Neurosurg 69: 332-339

9. Jennett B, Bond M (1975) Assessment of outcome after severe brain damage. Lancet (1975 March 1): 480-484 
10. Knuckey NW, Epstein MH, Haas R, Sparadeo F (1988) Distal anterior choroidal artery aneurysm: intraoperative localization and treatment. Neurosurgery 22 (6): 1084-1087

11. Lassen NA (1985) Cerebral blood flow tomography with Xenon133 Seminars in Nuclear Medicine 15(4): 347-356

12. Maurer AH (1988) Nuclear medicine: SPECT comparisons to PET. Radiology Clinics of North America 26 (5): 1059-1074

13. Merory J, Thomas DJ, Humphrey PRD, Du Boulay GH, Marshall J, Ross Russell RW, Symon L, Zilkha E (1980) Cerebral blood flow after surgery for recent subarachnoid haemorrhage. J Neurol Neurosurg Psychiatry 43: 214-221

14. Meyer CHA, Lowe D, Meyer M, Richardson PL, Neil-Dwyer $G$ (1983) Progressive change in cerebral blood flow during the first three weeks after subarachnoid hemorrhage. Neurosurgery 12: $58-76$

15. Mickey B, Vorstrup S, Voldby B, Lindewald $\mathrm{H}$, Harmsen A, Lassen NA (1984) Serial measurement of regional cerebral blood flow in patients with $\mathrm{SAH}$ using $133 \mathrm{Xe}$ inhalation and emission computerized tomography. J Neurosurg 60: 916-922

16. Obrist WD, Thompson HK, Wang HS, Wilkinson WE (1975) Regional cerebral blood flow estimated by $133-X$ enon inhalation. Stroke 6: 245-256

17. Pasqualin A, Kassel NF, Torner JC, Benedetti A, DaPian R, Guidetti B, Marini G, Mingrino S, Pluchino F, Testa C, Villani R (1988) Results of treatment. J Neurosurg Sci 32: 25-38

18. Robertson WM, Welch KMA, Tilley BC, Ewing JR (1988) Cerebral blood flow asymmetry in the detection of extracranial cerebrovascular disease. Stroke 19: 813-819
19. Rosenstein J, Suzuki M, Symon L, Redmond S (1984) Clinical use of a portable bedside cerebral blood flow machine in the management of aneurysmal subarachnoid hemorrhage. Neurosurgery $15(4)$ : 519-525

20. Rosenstein J, Wang ADJ, Symon L, Suzuki M (1985) Relationship between hemispheric cerebral blood flow, central conduction time, and clinical grade in aneurysmal subarachnoid hemorrhage. J Neurosurg 62: 25-30

21. Schroeder T, Holstein P, Lassen NA, Engell HC (1986) Measurement of cerebral blood flow by intravenous Xenon-133 technique and a mobile system. Neurol Res 8: 237-242

22. Shirahata N, Henriksen L, Vorstrup S, Holm S, Lauritzen $M$, Paulson OB, Lassen NA (1985) Regional cerebral blood flow assessed by $133 \mathrm{Xe}$ inhalation and emission tomography: normal values. J Comput Assist Tomogr 9: 861-866

23. Weir B, Menon D, Overton T (1978) Regional cerebral blood flow in patients with aneurysms: estimation by Xenon 133 inhalation. Can J Neurol Sci 5: 301-305

Correspondence and Reprints: James M. Mountz, M.D., Ph.D., Assistant Professor of Internal Medicine, The University of Michigan Medical Center, Division of Nuclear Medicine, Department of Intemal Medicine, B1G-412/0028 University Hospital, 1500 E. Medical Center Drive, Ann Arbor, MI 48109-0028, U.S.A. Present address: Associate Professor of Radiology, The University of Alabama at Birmingham, Division of Nuclear Medicine, 619 South $19^{\text {th }}$ Street, Birmingham, Al 35233, U.S.A. 